\title{
Lorentzian Algebra for the Superstring
}

\author{
V. Alan Kostelecký \\ Physics Department, Indiana University, Bloomington, Indiana 47405 \\ and \\ O. Lechtenfeld \\ Theoretical Division, CERN, Switzerland \\ (Received 9 February 1987)
}

\begin{abstract}
A Lorentzian algebra is constructed that underlies the covariant formulation of the spinning string. It is a simply laced hyperbolic Kac-Moody algebra having a realization on the ghost-extended spectrum of string states. From the weight space of this algebra, we obtain a unique indefinite superalgebra that has a realization on the superstring spectrum and that automatically implements the Gliozzi-Scherk-Olive projection.
\end{abstract}

PACS numbers: 11.17.+y, 02.20.+b, 11.30.Pb

One of the major quests in string theory is the search for symmetries underlying its remarkable properties. An important advance is the Frenkel-Kac realization ${ }^{1}$ of simply laced finite and affine Lie algebras in terms of bosonized vertex operators. Among other applications, this construction relates the spectrum of any ten-dimensional string to the weight lattice of an associated Lie algebra.

In the covariant formulation, ${ }^{2}$ bosonization of the fermionic ghost naturally leads to a Lorentzian extension of the weight lattice. ${ }^{3,4}$ This suggests the existence of a larger algebra, which for the superstring would incorporate covariant supersymmetry. In this Letter, we identify a particular Lorentzian algebra that is a key structure underlying string spectra. We also find a related indefinite superalgebra relevant to the superstring case.

Since the weight lattice is Lorentzian, we need an extension of the Frenkel-Kac construction. Consider an arbitrary integral indefinite lattice $\Gamma^{p, q}$ in $R^{p, q}$ with metric signature $(+)^{p}(-)^{q}$, generated by the $p+q$ basis vectors $e^{\mu}$. Introduce $p+q$ two-dimensional free massless scalar fields $\phi^{\mu}(z)$. Then, to each lattice point $\alpha=\alpha_{\mu} e^{\mu}$, associate an infinite tower of operators

$$
E_{(\zeta)}^{\alpha}=\oint \frac{d z}{2 \pi i} e_{(\zeta)}^{\alpha}(z):=\oint \frac{d z}{2 \pi i} \partial^{(\zeta)} \phi(z) e^{\alpha \cdot \phi}(z) c_{\alpha},
$$

where

$$
\begin{aligned}
& \partial^{(\zeta)} \phi(z)=\prod_{i=1}^{r} \partial^{\zeta_{i}} \phi^{\mu_{i}}(z), \quad r, \zeta_{i} \in N, \\
& \partial^{(0)} \phi(z):=1 .
\end{aligned}
$$

The cocycle operators $c_{\alpha}$ are given by

$$
\begin{gathered}
c_{\alpha}=\exp \left[i \pi\left(\alpha \cdot M \oint \frac{d z}{2 \pi i} \partial \phi\right)\right] \\
M_{v}^{\mu}= \begin{cases}0, & \mu \leq v \\
\pm 1, & \mu>v .\end{cases}
\end{gathered}
$$

For convenience, the Lorentz indices $\mu_{1}, \ldots, \mu_{r}$ associated to $(\zeta)=\left(\zeta_{1}, \ldots, \zeta_{r}\right)$ are suppressed. Normal ordering is understood throughout.

Eventually, we wish to identify the operators $E_{(\zeta)}^{\alpha}$ as vertex operators in a string theory. To do so, several constraints must be imposed. Conformal invariance relates $|\zeta|=\sum_{i} \zeta_{i}$ to $\alpha$ and selects certain linear combinations of the $E_{(\zeta)}^{\alpha}$. Furthermore, only a subset of the lattice is admissible because of physical constraints; examples of such constraints are $m_{L}^{2}=m_{R}^{2}$ and the restriction to specified spinor ghost sectors. Note also that the operators $E_{(\zeta)}^{\alpha}$ with $\alpha$ and $|\zeta|$ fixed are linearly dependent through the relations

$$
\oint \frac{d z}{2 \pi i} \partial f\left(\partial \phi, e^{\alpha \cdot \phi}\right)=0
$$

for arbitrary $f$.

The complete set $\left\{E_{(\zeta)}^{\alpha}\right\}$ may be given an algebraic structure by invoking operator-product expansions:

$$
\partial^{(\zeta)} \phi(z) e^{\alpha \cdot \phi}(z) \partial^{(\eta)} \phi(w) e^{\beta \cdot \phi}(w) \sim \sum_{n=\alpha \cdot \beta-|\zeta|-|\eta|}^{\infty}(z-w)^{n} \sum_{(\theta)}^{\prime} c_{(\zeta)(\eta)}^{n(\theta)}(\alpha, \beta) \partial^{(\theta)} \phi(w) e^{(\alpha+\beta) \cdot \phi}(w) .
$$

As before, Lorentz indices are suppressed; the prime indicates the restriction $|\theta|=|\zeta|+|\eta|-\alpha \cdot \beta+n$. Then, since the contour integrals select $n=-1$, we find for the nonvanishing graded commutators

$$
\left[E_{(\zeta)}^{\alpha}, E_{(\eta)}^{\beta}\right\}=\epsilon(\alpha, \beta) \sum_{(\theta)}^{\prime} c \overline{(\zeta)}_{(\eta)}^{1(\theta)}(\alpha, \beta) E_{(\theta)}^{a+\beta}
$$


where

$$
\left[E^{\alpha}, E^{\beta}\right\}:=E^{\alpha} E^{\beta}-(-1)^{\alpha^{2} \beta^{2} E^{\beta} E^{\alpha},}
$$

and

$$
\begin{aligned}
& \epsilon(\alpha, \beta):=\exp [i \pi(\alpha \cdot M \beta)] \\
& |\theta|=|\zeta|+|\eta|-\alpha \cdot \beta-1
\end{aligned}
$$

The choice (3) for the cocycle operators results in a superalgebra $Z_{2}$ grading in Eq. (6): Operators from even and odd points obey commutation and anticommutation rules, respectively. ${ }^{5}$

The structure of the algebra (6) may be partially understood as vector addition on the lattice, $\alpha+\beta \in \Gamma^{p, q}$. Note that the conformal dimensions of the operators in Eq. (6), given for $E_{(\zeta)}^{\alpha}$ by $h_{\alpha}=\frac{1}{2} \alpha(\alpha+Q)+|\zeta|-1$ for some background charge $Q$, satisfy $h_{\alpha}+h_{\beta}=h_{\alpha+\beta}$. The subalgebra $V$ of invariant or vertex operators, which has $h=0$, plays a dominant role. As any such operator is a conserved charge, the string states at any given mass level fall into representations of $V$. In the following, we focus on $V$ alone.
A Fock-space representation of the algebra (6) provides the link to the string spectrum. The vacuum $|0\rangle$ obeys $a_{n}^{\mu}|0\rangle=0$ for $n \geq 0$, where the $a_{n}^{\mu}$ are coefficients in the mode expansion

$$
\partial \phi^{\mu}(z)=\sum_{n=-\infty}^{+\infty} a_{n}^{\mu} z^{-n-1} .
$$

The state $|\alpha,(\zeta)\rangle$ is created from the vacuum by action of the integrand of Eq. (1) at $z=0$ :

$$
|\alpha,(\zeta)\rangle=e_{(\zeta)}^{\alpha}(0)|0\rangle .
$$

The algebra $V$ is a direct generalization of the standard vertex-operator realization of Lie algebras. As usual, the Cartan subalgebra of $V$ is spanned by the momentum operator $E_{(1)}^{0}=a_{0} \equiv p$ :

$$
\begin{aligned}
& {\left[p^{\mu}, E_{(\zeta)}^{\alpha}\right]=\alpha^{\mu} E_{(\zeta)}^{\alpha},} \\
& {\left[E_{(\zeta)}^{\alpha}, E_{(\eta)}^{-\alpha}\right\}=\epsilon(\alpha,-\alpha) c_{(\zeta)(\eta)}^{-1(1)} \cdot p \delta_{\alpha^{2}+|\zeta|+|\eta|, 2} .}
\end{aligned}
$$

Finite-dimensional subalgebras of $V$ occur only for $\alpha$ restricted to a Euclidean sublattice $(q=0)$ and $\alpha^{2} \leq 2$, as may be seen from the graded commutators

$$
\left[E_{(0)}^{\alpha}, E_{(0)}^{\beta}\right\}=\epsilon(\alpha, \beta) \times\left\{\begin{array}{l}
0, \quad \alpha \cdot \beta \geq 0 \\
E_{(0)}^{\alpha+\beta}, \quad \alpha \cdot \beta=-1, \\
\frac{1}{2}(\alpha-\beta) \cdot E_{(1)}^{\alpha+\beta}, \quad \alpha \cdot \beta=-2, \\
-\frac{1}{2}(\alpha \times \beta) \cdot E_{(1,1)}^{\alpha+\beta}, \quad \alpha \cdot \beta=-3, \\
\cdots
\end{array}\right.
$$

Thus, for $\Gamma^{p, 0}$ we obtain simply laced Lie algebras and superalgebras. ${ }^{6}$ In contrast, the bosonized covariant formulation of the spinning string requires $q=1$. The resulting algebras, called Lorentzian, are indefinite KacMoody algebras ${ }^{7}$ or their superalgebra generalizations.

Modular invariance of string theories requires that the relevant lattices be self-dual. ${ }^{8}$ In the Lorentzian case, such lattices are determined by their dimension, $p+1$. The only even self-dual Lorentzian lattices are

$$
\Pi^{4 n+1,1}:=\left\{\alpha \text { or } \alpha-\lambda \in Z^{4 n+1,1} \mid \alpha \cdot \lambda \in Z\right\},
$$

where $\lambda$ is a constant vector with components $\lambda^{\mu}=\frac{1}{2}$, and $n$ is even. Furthermore, any odd self-dual Lorentzian lattice is isomorphic to $Z^{p+1,1}$, modulo a Lorentz rotation. ${ }^{9}$

An example important for string theory is the odd self-dual lattice $\Pi^{4 n+1,1}$, where $n$ is $o d d$. This lattice can be so $(4 n+1,1)$-rotated into $Z^{4 n+1,1}$. Note, however, that these two lattices can lead to physically inequivalent theories because the condition $h=0$ is not Lorentz invariant in the presence of a background charge $Q$.

Both $\Pi^{4 n+1,1}$ and $Z^{4 n+1,1}$ are embedded in the nonin- tegral lattice

$$
\begin{aligned}
\Lambda^{p, 1} & =\left\{\alpha \text { or } \alpha-\lambda \in Z^{p, 1}\right\}, \quad p=4 n+1, \\
& =(o) \cup(v) \cup(s) \cup(c),
\end{aligned}
$$

as the conjugacy classes $(o) \cup(s)$ and $(o) \cup(v)$, respectively. The conjugacy-class addition table and scalar products are identical to those of so $(8 n)$. This larger lattice can be interpreted as the weight lattice of a Lorentzian algebra, $D_{p, 1}$, whose root lattice is the conjugacy class $(o)$. The latter is spanned by the simple roots $\alpha^{i}$ of so $(2 p) \equiv D_{p}$ and by an extra root $\alpha^{0}$ :

$$
\begin{aligned}
& \alpha^{i}=e^{i}-e^{i+1}, \quad i=1, \ldots, p-1, \\
& \alpha^{p}=e^{p-1}+e^{p}, \\
& \alpha^{0}=e^{0}-e^{1}-e^{2}-e^{3} .
\end{aligned}
$$

The corresponding Dynkin diagram is shown in Fig. 1. The algebra may also be obtained from the affine algebra $D_{p-1}^{(1)}$ by addition to the root lattice of a second null direction. 


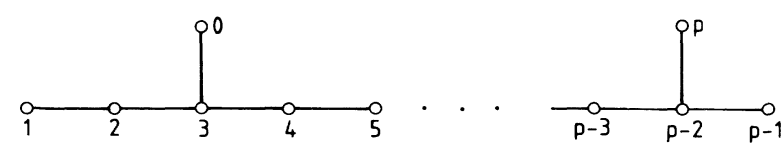

FIG. 1. Dynkin diagram for $D_{p, 1}$.

For the ten-dimensional string theories with $p=5$, $q=1, \Gamma^{5,1}$ should be the minimal Lorentzian extension of the weight lattice of $\operatorname{so}(10) \equiv D_{5}$, which is $\Lambda^{5,1}$. The associated Lorentzian algebra is $D_{5,1}$, whose Dynkin diagram, shown in Fig. 2, exhibits a triality symmetry because the elementary vector and spinor weights have the same length. It is the unique simple simply laced Lorentzian extension of so(10) and is a hyperbolic KacMoody algebra. ${ }^{10}$

The algebra $D_{5,1}$ governs the space-time part of the ghost-extended spinning-string spectrum, as may be seen from its elementary weights and the fields that create them from the vacuum:

$$
\begin{aligned}
& \lambda_{o}=(10000,-1) \sim \psi(-1), \\
& \lambda_{v}=(00000,-1) \sim T_{(-1)} \text {, } \\
& \lambda_{s}=\frac{1}{2}\left(\begin{array}{lllll}
1 & 1 & 1 & 1 & 1,-3) \sim S_{(-3 / 2)},
\end{array}\right. \\
& \lambda_{c}=\frac{1}{2}(111111-1,-3) \sim \dot{S}_{(-3 / 2)} .
\end{aligned}
$$

Here, $\psi$ is the Neveu-Schwarz-Ramond field, $T$ is the tachyon of the bosonic string, and $S, \dot{S}$ are spin fields of opposite helicity. The subscript in parentheses indicates the ghost charge $q .^{4}$

It is intriguing that $D_{5,1}$ automatically yields the correct correlation between the ghost charges and the so(10) conjugacy classes. Moreover, with $q=-1$ in the Neveu-Schwarz sector and $q=-\frac{3}{2}$ in the Ramond sector, the algebra naturally selects one of the two canonical choices for the spinor ghost sectors.

Note that to obtain an integral lattice $\Lambda^{5,1}$ must be projected either to $Z^{5,1}$ or to $\Pi^{5,1}$. The former possibility restricts the spectrum to the Neveu-Schwarz sectors of the spinning string and is possible in any even dimension. The latter yields the superstring and is possible only for dimensionality $8 n+2$.

The role of supersymmetry has not yet been directly addressed. One approach is to find an integral sublattice of $\Lambda^{5,1}$ that plays the role of a root lattice for some superalgebra. This may be achieved by extending the root lattice of $D_{5,1}$ by an odd weight in the $(v),(s)$, or $(c)$ conjugacy classes. Since a minimal simple extension is desired, any roots that become nonsimple must be removed. The procedure is analogous to the construction of $E_{8}$ from the so(16) root lattice by addition of the conjugacy class $(s)$.

Addition of a vector weight $e^{5}$ and removal of $\alpha^{5}$ results in a root lattice $Z^{5,1}$, while addition of a spinor weight $-2 e^{0}-\lambda_{s}$ and removal of $\alpha^{0}$ yields $\Pi^{5,1}$. The

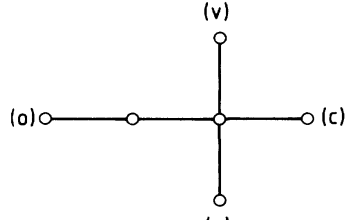

(s)

FIG. 2. Dynkin diagram for $D_{5,1}$.

associated superalgebra $E_{5,1}$ is the unique simple simply laced extension of $D_{5,1}$. It can be minimally represented by the Dynkin-Kac diagram ${ }^{11}$ of Fig. 3.

Supersymmetry generators are invariant operators associated to odd points $\alpha$ with $\alpha^{2} \leq 1$. For the NeveuSchwarz sectors of the spinning string, $(x)=(v)$, the anticommuting generators in the algebra $E_{5,1}$ have $h \in Z$ $+\frac{1}{2}$ and so are not conserved. They change the level number $N$ by odd half-units and map Gliozzi-ScherkOlive (GSO)-accepted states into GSO-rejected ones. For the superstring, $(x)=(s)$, the anticommuting generators have $h \in Z$ since $Q=2 e^{0}$. The subset corresponding to lattice points satisfying $\frac{1}{2} \alpha^{2}-q \leq 1$ is a set of conserved supersymmetry charges. All generators commute with the GSO projection.

Note that $D_{5,1}$ and $E_{5,1}$ are the invariant subalgebras $V$ of the full lattice algebras associated with $Z^{5,1}$ and $\Pi^{5,1}$, respectively.

The maximal finite subsuperalgebra of $E_{5,1}$ is the semidirect sum of a Grassmann algebra with su(5). For the superstring, this results in a partial symmetry on the spectrum. No finite superalgebra exists with a nontrivial action on the whole superstring spectrum. This suggests that an off-shell realization of supersymmetry will involve an infinite number of auxiliary fields.

In addition to their intrinsic interest as extensions of the usual string-spectrum symmetry in which the ghostextended spin fields arise naturally, the lattice algebras $D_{5,1}$ and $E_{5,1}$ have other consequences. For example, the picture-changing operation, ${ }^{2}$ which relates different slices of the weight lattice of $E_{5,1}$, is expected to have an algebraic realization. More computationally useful applications could include the use of the Ward identities of $D_{5,1}$ to compute correlations by the reduction technique. ${ }^{12}$ Furthermore, enlargements of the algebras may be significant. Extension to the heterotic strings ${ }^{8}$ could yield further insights on their origin and relationships, while inclusion of the space-time operators $\partial X$ might

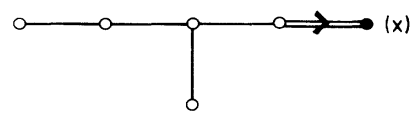

FIG. 3. Dynkin-Kac diagram for $E_{5,1}$. The symbol $(x)$ represents the possible conjugacy classes $(v),(s)$, or $(c)$ in $D_{5,1}$ of the labeled root. 
lead to an improved understanding of the nature of space-time supersymmetry.

We thank V. Deodhar and S. Samuel for discussion. One of us (V.A.K.) acknowledges support of the United States Department of Energy under Contract No. DEAC02-84ER40125, Task B.

${ }^{1}$ I. Frenkel and V. G. Kac, Invent. Math. 62, 23 (1980); G. Segal, Commun. Math. Phys. 80, 301 (1981).

${ }^{2}$ See, for example, D. Friedan, E. Martinec, and S. Shenker, Nucl. Phys. B271, 93 (1986), and references therein.

3 J. Cohn, D. Friedan, Z. Qui, and S. Shenker, Nucl. Phys. B278, 577 (1986).

${ }^{4}$ V. A. Kostelecký, O. Lechtenfeld, W. Lerche, S. Samuel, and S. Watamura, Nucl. Phys. B288, 173 (1987).

${ }^{5}$ For covariance of the structure constants, define $M$ as in Ref. 4.
${ }^{6} \mathrm{We}$ disregard here the world-sheet dependence of the vertex operators. The associated higher modes may be incorporated by addition to $\Gamma^{p, q}$ of an orthogonal null dimension. For Euclidean lattices, $q=0$, this enlarges the algebra to its affine extension; see P. Goddard and D. Olive, in Vertex Operators in Mathematics and Physics, edited by J. Lepowsky, S. Mandelstam, and I. M. Singer (Springer-Verlag, Berlin, 1984), p. 51. We also disregard here the space-time operators $\partial X$, whose action further extends the algebra.

${ }^{7}$ V. G. Kac, Funct. Anal. Appl. 1, 328 (1967); R. V. Moody, Bull. Am. Math. Soc. 73, 217 (1967).

${ }^{8}$ D. J. Gross, J. A. Harvey, E. Martinec, and R. Rohm, Nucl. Phys. B256, 253 (1985), and B267, 75 (1986).

${ }^{9}$ See Goddard and Olive in Ref. 6.

${ }^{10}$ R. V. Moody, Adv. Math. 33, 144 (1979); A. P. Ogg, Can. J. Math. 36, 800 (1984).

${ }^{11}$ V. G. Kac, Adv. Math. 26, 8 (1977).

${ }^{12} \mathrm{~S}$. Samuel, City College, City University of New York, Report No. CCNY-HEP-86/15, 1986 (to be published). 